(C) 2008 The Japan Society of Applied Physics

\title{
Alignment Control of Liquid Crystals Using Conductive Atomic Force Microscopy Nanolithography
}

\author{
Tzu-Chieh LIN* and Chih-Yu CHAO \\ Department of Physics, National Taiwan University, Taipei 10617, Taiwan, Republic of China
}

(Received January 29, 2008; revised March 3, 2008; accepted March 9, 2008; published online June 13, 2008)

Conductive atomic force microscopy (CAFM) nanolithography was used to modify a silicon surface. This approach generating the silicon oxide grating by CAFM gives a control of liquid crystal (LC) alignment in the micron or submicron region. It establishes a pixel with a smaller size to achieve high-resolution images. Compared with the conventional cloth rubbing and AFM scratching techniques, the CAFM nanolithography prevents scratching damage, dust contamination and residual static electricity problems. Furthermore, this inorganic alignment method can also avoid the damage caused by UV light exposure and high-temperature environment. [DOI: 10.1143/JJAP.47.4583]

KEYWORDS: liquid crystals, atomic force microscopy

\section{Introduction}

To achieve high-resolution liquid crystal displays (LCDs), alignment control of liquid crystal (LC) plays a significant role. In the 1990's, Shigeno et al. utilized scanning tunneling microscopy (STM) to observe the configuration of LC on the graphite surface. ${ }^{1)}$ Concerning the interaction energy between the substrate and molecules, they constructed models for liquid crystal arrangement. For LCDs, the main mechanism responsible for azimuthal LC alignment is proposed to be surface grooves based on Berreman's continuum theory. ${ }^{2)}$ For nowadays mass production, conventional rubbing by cloth is widely used for LC alignment. But cloth rubbing is insufficient to dominate the LC orientation in the micron or submicron domain. To improve this drawback, Rüetschi et al. scratched the polymer surface by atomic force microscopy (AFM). ${ }^{3)}$ More than successfully aligned LCs, they developed an optical waveguide of one low effective refractive index in the middle of two high index domains resulting from two different AFM writing directions. There are many other researches using the AFM scratching technique for LC alignment in the past few years. ${ }^{4-7)}$ Wen et al. fabricated the LC cell with gray scale by rotating the AFM scratching direction across the adjacent stripe regions. ${ }^{5}$ Kim et al. used the AFM stylus to perform an in-plane surface multistability due to the multi-fold rotational symmetry of the pattern. ${ }^{6)}$ They also proposed that LC pretilt angle is a function of line density scratched by AFM. ${ }^{7)}$ However, both of cloth rubbing and AFM scratching techniques may produce scratching damage, dust contamination and residual static electricity on the polyimide (PI) substrates. In addition, the scratching method is quite harmful to the stylus of AFM. In this letter, we demonstrate a method using conductive AFM (CAFM) to generate periodical $\mathrm{SiO}_{2}$ grating on a doped silicon surface for LC alignment. $^{8-10)}$ The CAFM nanolithography enables us to govern precisely the direction and the line separation of the $\mathrm{SiO}_{2}$ grating as it is required. We find this approach generating the silicon oxide grating by CAFM can anchor the directions of LCs on the substrate and gives a control of LCs alignment in the micron or submicron region. According to the Frank elastic energy theory, the global configuration of LC direction is parallel to the surface-relief

*E-mail address: tclin@phys.ntu.edu.tw grooves to minimize orientational distortion. ${ }^{11)}$ Moreover, this inorganic LC alignment method using CAFM not only avoids the drawbacks caused by conventional cloth rubbing and AFM scratching techniques, but also establishes a pixel with a smaller size to achieve high-resolution images. Furthermore, unlike PI alignment layer, the inorganic LC alignment method by CAFM can avoid damage generated by long-time UV light exposure and high-temperature environment in LCD projector.

\section{Experiments}

\subsection{CAFM nanolithography}

In our experiments, the silicon wafers used for AFM writing were p-type (resistivity $1-20 \Omega \mathrm{cm}$ ), and oriented in (100) direction. This wafer is conductible and polished so as to be used as a reflector. Thus a parallax problem occurring when the reflector is placed behind the rear substrate could be averted. ${ }^{12)}$ The wafers were cleaned by ethanol in an ultrasound cleaner for $5 \mathrm{~min}$ and subsequently rinsed by deionized water. After cleaning, for H-passivated surface, the samples were dipping in $10 \%$ HF solution by $60 \mathrm{~s} .^{8,9)}$ The wafers were cleaned again by the above steps and purged by nitrogen gas. Then the surface preparation was completed for CAFM writing. CAFM nanolithography was carried out in air using Veeco CP-R microscope with the AFM tip (Ultrasharp) coated with $\mathrm{Cr} / \mathrm{Au}$ conductive film and operated in the contact mode. In writing $\mathrm{SiO}_{2}$ pattern, the protruding areas were observed when a positive bias was applied on the silicon substrate at a voltage above $5 \mathrm{~V}$ and the AFM tip was grounded. Figure 1 represents the architecture of CAFM system. This nanolithography utilizes a thin layer of absorbed water covering on the sample and the tip. ${ }^{13)}$ The CAFM nanolithography was performed at the speed $1 \mu \mathrm{m} / \mathrm{s}$ with the density of 2.5 lines $/ \mu \mathrm{m}$. All the lithography process was done at room temperature with an ambient relative humidity of approxmiately $60 \%$. The topography image of $\mathrm{SiO}_{2}$ line pattern was taken by AFM at scanning rate of $6 \mu \mathrm{m} / \mathrm{s}$ with the same tip when zero voltage was applied to the silicon substrate [see Fig. 2(a)]. As shown in Fig. 2(b), the substrate bias of $7 \mathrm{~V}$ gives oxidation width of $250 \mathrm{~nm}$ and height of $7 \mathrm{~nm}$ approximately. Figure 2(c) taken by scanning electron microscope (SEM) shows the $\mathrm{SiO}_{2}$ grating generated at bias voltage of $8 \mathrm{~V}$. The variation of oxidation height $h$ vs voltage $V$ is demonstrated in Fig. 3. The height of oxidation increases with the applied bias voltage. 


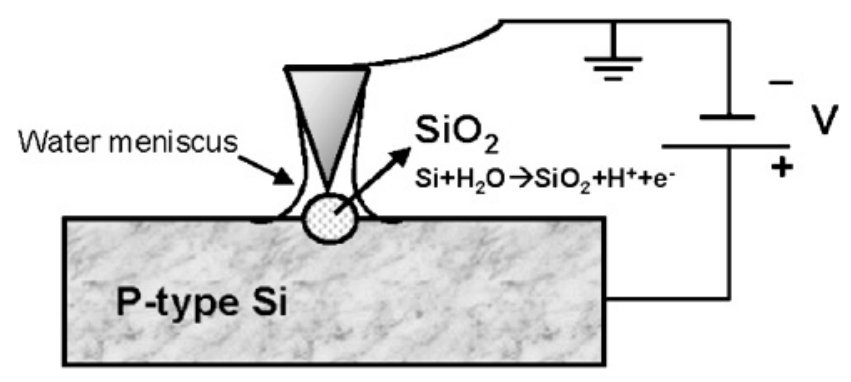

Fig. 1. Schematic of the CAFM system. In order to generate silicon oxide patterns, the p-type silicon substrate is applied by a positive bias and the AFM tip is grounded.

(a)

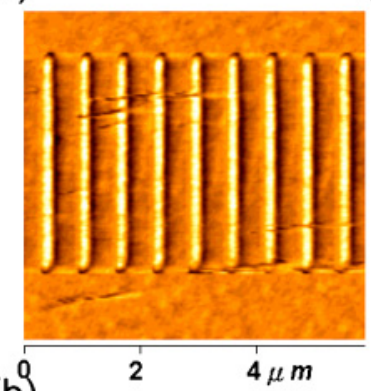

(b)

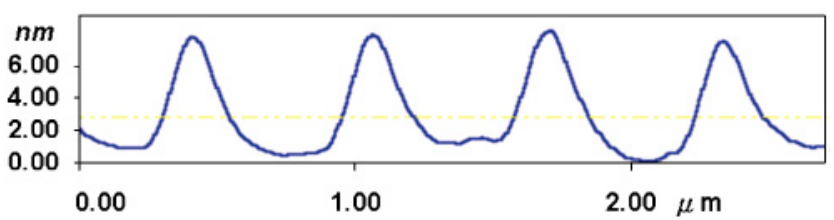

Fig. 2. (Color online) (a) The AFM image of $\mathrm{SiO}_{2}$ grating line pattern written by applying the substrate under a $+7 \mathrm{~V}$ bias voltage with the writing speed of $1 \mu \mathrm{m} / \mathrm{s}$ at room temperature and relative humidity $\sim 60 \%$. This topography image was taken at scanning rate of $6 \mu \mathrm{m} / \mathrm{s}$ when zero voltage was applied to the silicon substrate. (b) The height profile of a $\mathrm{SiO}_{2}$ grating line. The substrate bias of $7 \mathrm{~V}$ gives oxidation width of full width at half maximum (FWHM) $250-280 \mathrm{~nm}$ and height of $7 \mathrm{~nm}$ approximately. (c) SEM image of $\mathrm{SiO}_{2}$ grating generated at substrate bias of $8 \mathrm{~V}$. The pattern line width is about $300 \mathrm{~nm}$.

\subsection{Fabrication and measurements of LC cells}

The silicon substrate patterned by CAFM was placed opposite the conventionally rubbed PI layer which was grown on top of an indium tin oxide (ITO) coated glass substrate. Then, we filled the empty cell with a 4- $n$-pentyl4 -cyanobiphenyl (5CB) LC at room temperature. The PI rubbing direction on the counter surface was set orthogonal to the CAFM writing direction. Therefore, the director configuration of LCs sandwiched between the $\mathrm{SiO}_{2}$ patterned substrate and rubbed PI layer became $90^{\circ}$ twisted and the background was homogeneous. The cells are inspected by a polarizing optical microscopy in the reflection mode and the polarizer setting is demanded to obtain maximum brightness with zero voltage between the substrates (see Fig. 4). The patterned area of the cell belongs to normal white. The bright region has size of $10 \times 20 \mu \mathrm{m}^{2}$ as shown in Fig. 4. The polarizer setting makes the polarizer and the LC director near the upper substrate not parallel but with some angle to form a mixed-mode twisted nematic (MTN) LC cell. In direct-view display, the MTN LC cell requires only one front polarizer to prevent the parallax.

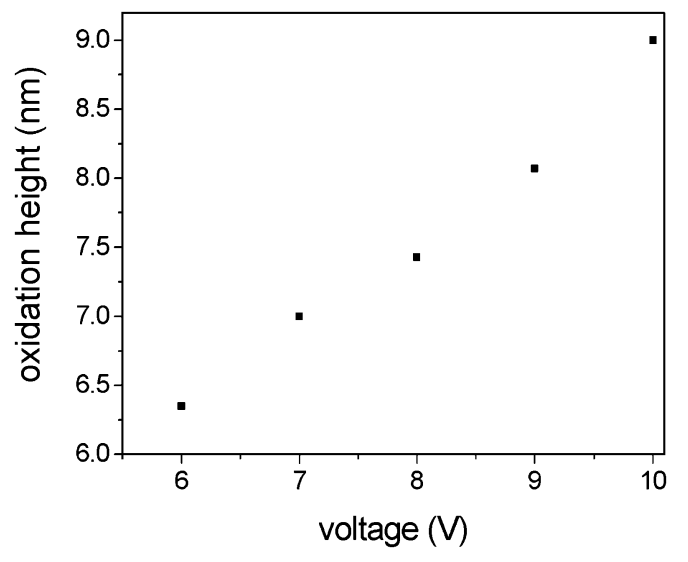

Fig. 3. The variation of oxidation height $h$ vs voltage $V$ (the substrate is positively biased with respected to the tip). The height of oxidation increases with the applied bias voltage.

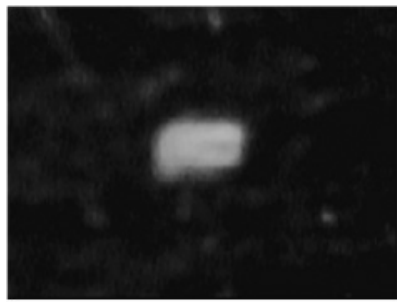

(a)

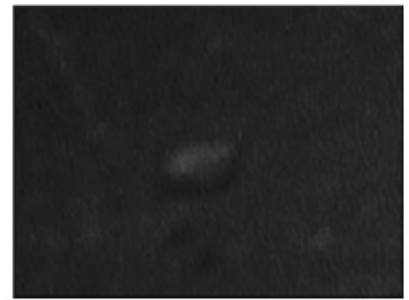

(b)
Fig. 4. Polarizing optical micrograph of the reflective LC cell. The bright region has size of $10 \times 20 \mu \mathrm{m}^{2}$. (a) The bright state with zero voltage. (b) The dark state with voltage at $7.5 \mathrm{~V}$.

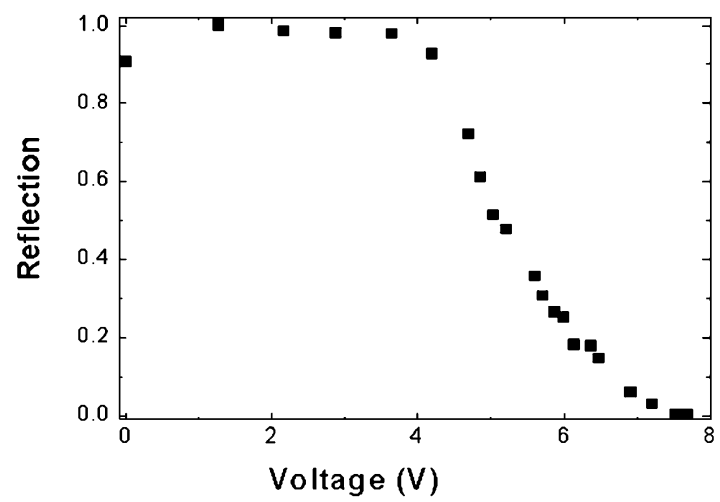

Fig. 5. The voltage-dependent light reflectance of a MTN LC cell. The LC cell switches to its gray scale when the applied voltage is above $4 \mathrm{~V}$. Increasing the voltage to $\sim 7.5 \mathrm{~V}$, the cell becomes dark.

Utilizing the conductivity of the doped silicon, the p-type silicon we used here was not only to be an anode but also a driving electrode. The doped and polished silicon substrate patterned by CAFM can thus be simultaneously used for LC alignment, cell electrode, and mirror reflector. Applying the voltage between the ITO and p-type silicon substrates above $4 \mathrm{~V}$, the patterned area of the cell, i.e., normally white mode, started to change its phase retardation. While the applied voltage was increased, the image became darker as shown in Fig. 4. Figure 5 demonstrates the voltage-dependent light reflectance of the reflective MTN LC cell. At voltage around $7.5 \mathrm{~V}$, the phase retardation came into $\pi$ resulting in the cell being the black state. Based on Berreman's groove model, ${ }^{2)}$ 
the contribution of morphology to anchoring energy is given by $W=2 \pi^{3} A^{2} K / \lambda^{3}$, where $A$ is the amplitude, $\lambda$ is the pitch of the surface corrugation, and $K$ is the Frank elastic constant of the LC. With $A \sim 7 \mathrm{~nm}, \lambda \sim 400 \mathrm{~nm}$, and $K_{5 \mathrm{CB}}=2 \times 10^{-12} \mathrm{~N}$, we obtain the anchoring energy of $\mathrm{SiO}_{2}$ grating is $94.8 \times 10^{-9} \mathrm{~N} / \mathrm{m}\left(\sim 10^{-6} \mathrm{~N} / \mathrm{m}\right)$ which is in the same order of AFM rubbing result. ${ }^{7)}$

\section{Conclusions}

In conclusion, we have shown that the silicon surfacerelief grating of $\mathrm{SiO}_{2}$ by CAFM could anchor LC molecules on the substrate in order directly. The CAFM nanolithography could reduce the size and separation of the pixels in micron or submicron scale to obtain higher resolution for future LCD application. More importantly, this inorganic LC alignment method by CAFM not only prevents scratching damage, dust contamination and residual static electricity caused by conventional cloth rubbing and AFM scratching techniques, but also avoids damage generated by long-time UV light exposure and high-temperature environment in LCD projector. Finally, employing massive parallel AFM nanolithography with 55,000 styli proposed by Mirkin et al. ${ }^{14)}$ makes high throughput and shorter writing time possible in the future LCD application.

\section{Acknowledgments}

This work was supported by the National Science Council and Ministry of Education of the Republic of China.

1) M. Shigeno, W. Mizutani, M. Suginoya, M. Ohmi, K. Kajimura, and M. Ono: Jpn. J. Appl. Phys. 29 (1990) L119.

2) D. W. Berreman: Phys. Rev. Lett. 28 (1972) 1683.

3) M. Rüetschi, P. Grütter, J. Fünfschilling, and H.-J. Güntherodt: Science 265 (1994) 512.

4) A. J. Pidduck, S. D. Haslam, G. P. Bryan-Brown, R. Bannister, and I. D. Kitely: Appl. Phys. Lett. 71 (1997) 2907.

5) B. Wen, M. P. Mahajan, and C. Rosenblatt: Appl. Phys. Lett. 76 (2000) 1240 .

6) J. H. Kim, M. Yoneya, and H. Yokoyama: Nature 420 (2002) 159.

7) J. H. Kim, M. Yoneya, J. Yamamoto, and H. Yokoyama: Nanotechnology 13 (2002) 133.

8) L. Tsau, D. Wang, and K. L. Wang: Appl. Phys. Lett. 64 (1994) 2133.

9) D. Wang, L. Tsau, and K. L. Wang: Appl. Phys. Lett. 65 (1994) 1415.

10) K. Matsumoto: Oyo Buturi 67 (1998) 176 [in Japanese].

11) F. C. Frank: Discuss. Faraday Soc. 25 (1958) 19.

12) S. T. Wu and D. K. Yang: in Reflective Liquid Crystal Displays, ed. A. C. Lowe (Wiley-SID, Chichester, U.K., 2001) p. 89.

13) H. Sugimura and N. Nakagiri: Jpn. J. Appl. Phys. 34 (1995) 3406

14) K. Salaita, Y. Wang, J. Fragala, R. A. Vega, C. Liu, and C. A. Mirkin: Angew. Chem., Int. Ed. 45 (2006) 1. 